\title{
Linear and Sliding-Mode Controllers for Three-Phase Four-Wire Power Converters
}

\author{
VASCO SOARES ${ }^{1}$, PEDRO VERDELHO $^{2}$ \\ ${ }^{1}$ DEEA - Departamento de Engenharia Electrotécnica e Automação, ${ }^{1,2}$ DEEC-CAUTL \\ ${ }^{1}$ ISEL - Instituto Superior de Engenharia de Lisboa, ${ }^{2}$ IST - Instituto Superior Técnico \\ ${ }^{1}$ Rua Conselheiro Emídio Navarro, 1; 1950-062 Lisboa \\ PORTUGAL
}

\begin{abstract}
This paper presents the voltage regulation system design for a three-phase four-wire voltage converter using linear and sliding-mode control techniques. The regulation system is applied to a four-leg voltage source converter connected to the ac mains. System modelling and controllers syntheses are realized and compared. Digital simulations highlight the voltage regulation characteristics in terms of steady-state and transient responses. The robustness to disturbances influence is also analysed. Conclusions are taken with respect to the different approaches presented and the type of the application where the dc voltage regulation is needed, like active power filters, unified power flow controllers, neutral current compensators, pwm rectifiers and others.
\end{abstract}

Key-Words: Linear Control, Sliding-Mode Control, Voltage Regulation, Power Converter, Three-Phase Four-Wire Voltage Source Converter.

\section{Introduction}

Voltage regulation systems play an important role in all self-powered systems connected to the ac mains, like active filters (AF's), unified power flow controllers (UPFC's), advanced static VAr compensators (ASVC's), PWM rectifiers, uninterruptible power supplies (UPS's) and neutral current compensators (NCC's). In these converters the voltage regulation it is absolutely necessary to the operation of the voltage source converters (VSC) in order to sink or feed current into mains. Some works have been realized in the design of voltage regulators for three-phase four-wire converters [1]-[4]. An insight study of the voltage regulation system using a linear controller was already done [4], but less attention has been paid to sliding-mode controllers for power converters with neutral current control. In this paper a comparative study between linear and sliding-mode controllers is presented. Digital simulation results are shown to highlight the characteristics of both controllers with respect to dynamic and steady-state behaviour.

\section{Modelling}

The voltage regulation control system is based on a three-phase four-wire VSC shown in Fig. 1. The power converter is a four-leg VSC with the neutral wire connected to the fourth leg.

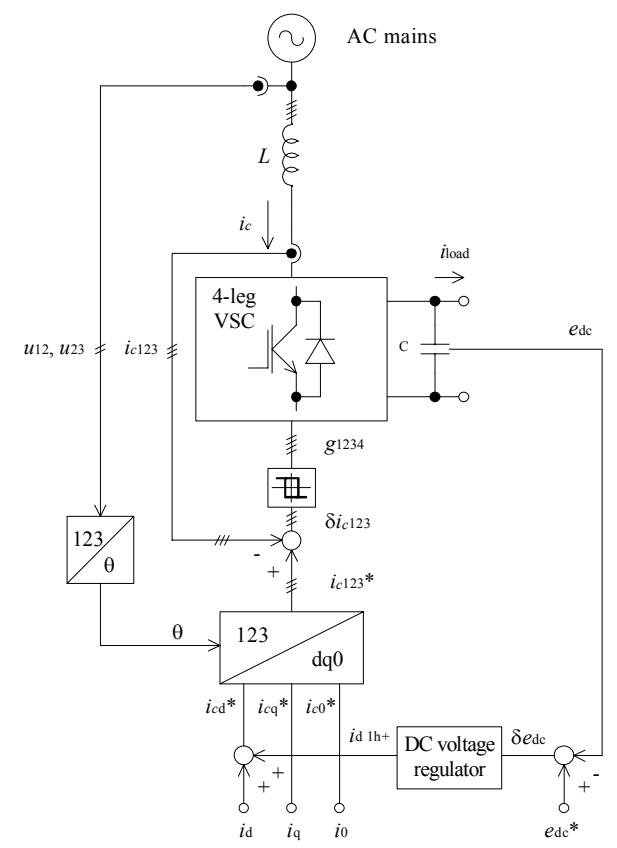

Fig. 1. Voltage regulation control system based on the four-leg VSC.

System dynamics of the connection mains-VSC can be established by state equations in vector-matrix form as in [4]. By Fig. 1 and using the Park's transformation, 
the state equation for the capacitor voltage (VSC dc side voltage) is,

$$
\dot{e}_{\mathrm{dc}}=\frac{i_{\mathrm{dc}}}{C}-\frac{i_{\text {load }}}{C}=\frac{f_{\mathrm{d}} i_{c_{\mathrm{d}}}+f_{\mathrm{q}} i_{c_{\mathrm{q}}}+f_{0} i_{c_{0}}}{C}-\frac{i_{\text {load }}}{C},
$$

where $f_{\mathrm{d}}, f_{\mathrm{q}}$ and $f 0$ are auxiliary switching functions. The system model in stationary coordinates dq0 [2] is presented (2).

$$
\begin{aligned}
& {\left[\begin{array}{c}
i_{c_{\mathrm{d}}} \\
\dot{i}_{c_{\mathrm{q}}} \\
\dot{i}_{c_{0}} \\
\dot{e}_{\mathrm{dc}}
\end{array}\right]=\left[\begin{array}{cccc}
-\frac{R}{L} & \dot{\theta} & 0 & -\frac{f_{\mathrm{d}}}{L} \\
-\dot{\theta} & -\frac{R}{L} & 0 & -\frac{f_{\mathrm{q}}}{L} \\
0 & 0 & -\frac{R+3 R_{\mathrm{N}}}{L+3 L_{\mathrm{N}}} & -\frac{f_{0}}{L+3 L_{\mathrm{N}}} \\
\frac{f_{\mathrm{d}}}{C} & \frac{f_{\mathrm{q}}}{C} & \frac{f_{0}}{C} & 0
\end{array}\right] \cdot\left[\begin{array}{c}
i_{c_{\mathrm{d}}} \\
i_{c_{\mathrm{q}}} \\
i_{c_{0}} \\
e_{\mathrm{dc}}
\end{array}\right]} \\
& +\left[\begin{array}{ccccc}
1 / L & 0 & 0 & 0 \\
0 & 1 / L & 0 & 0 \\
0 & 0 & 1 /\left(L+3 L_{\mathrm{N}}\right) & 0 \\
0 & 0 & 0 & -1 / C
\end{array}\right] \cdot\left[\begin{array}{c}
u_{\mathrm{d}} \\
u_{\mathrm{q}} \\
u_{0} \\
i_{\text {load }}
\end{array}\right]
\end{aligned}
$$

Active power is mainly dependent of current component $i_{c \mathrm{~d}}$. This current component controls the active power flow in the VSC and thus the capacitor voltage $e_{\mathrm{dc}}$. Neglecting the losses in inductances and switching devices,

$$
u_{\mathrm{d}} i_{c_{\mathrm{d}}}+u_{0} i_{c_{0}} \approx e_{\mathrm{dc}} i_{\mathrm{dc}} .
$$

By (1) and (3) the state equation for the capacitor voltage is now,

$$
\dot{e}_{\mathrm{dc}}=\frac{u_{\mathrm{d}} i_{c_{\mathrm{d}}}+u_{0} i_{c_{0}}}{C e_{\mathrm{dc}}}-\frac{i_{\text {load }}}{C} .
$$

\section{System Control with a Linear Controller}

The generic voltage regulation control system was already shown in Fig. 1. Currents $i_{c 123}{ }^{*}$ are obtained from $i_{c \mathrm{dq} 0}{ }^{*}$ by a Park's transformation presented in [2], [4], [5]. They derive from the input currents $i_{\mathrm{dq} 0}$ and also from the dc voltage regulation system. Current control is accomplished by four two-level hysteretic comparators operating in phase coordinates. Direct (active), quadrature (reactive) and zero sequence current components can be controlled by the input values $i_{\mathrm{dq} 0}$, depending in what kind of application the VSC is used (e.g. harmonics currents for AF proposes). A proportional-integral (PI) controller performs the voltage regulation in the VSC dc side. Its input is the capacitor voltage error $\delta e_{\mathrm{dc}}=e_{\mathrm{dc}}{ }^{*}-e_{\mathrm{dc}}$. Through the regulation of the first harmonic active current of positive sequence $i_{c \mathrm{dlh}}{ }^{+}$it is possible to control the active power flow in the VSC and thus the capacitor voltage $e_{\mathrm{dc}}$. It's worthwhile to note that the controller will not only feed the de load but also should handle the power dissipated in inductances and the losses in the switching devices. To obtain a linear regulator (4) should be linearized. The linearization and the PI synthesis were already realized in other papers [2]-[4]. The PI controller has a control law given by (5) where the variables $k_{\mathrm{P}}$ and $k_{\mathrm{I}}$ are the proportional and integral gains, respectively.

$$
i_{\mathrm{d}_{\mathrm{hh}}}^{+}=i_{c_{\mathrm{d}}}^{*}=k_{\mathrm{p}}\left(e_{\mathrm{dc}}^{*}-e_{\mathrm{dc}}\right)+k_{\mathrm{I}} \int\left(e_{\mathrm{dc}}^{*}-e_{\mathrm{dc}}\right) \mathrm{d} t
$$

Considering the equilibrium situation where an approximately null active power is flowing into the converter, i.e., $i_{c \mathrm{~d}}{ }^{\circ}=0$, the PI parameters are,

$$
k_{\mathrm{P}}=\frac{2 C e_{\mathrm{dc}}}{u_{\mathrm{d}}} \zeta \omega_{n} \quad, \quad k_{\mathrm{I}}=\frac{C e_{\mathrm{dc}}}{u_{\mathrm{d}}} \omega_{n}^{2} .
$$

A first order low-pass (LP) filter with a cutoff frequency $k_{\mathrm{I}} / k_{\mathrm{P}}$ is used to eliminate the influence of the zero introduced by the PI controller in the forward-path transfer-function (TF). The parameters considered in the controller synthesis for the resulting prototype second-order $\mathrm{TF}$ are: damping ratio $\zeta=\sqrt{ } 2 / 2=0.707$ (Butterworth or ITAE criteria for second-order systems), natural undamped frequency $\omega_{n}=\omega / 5=62.83 \mathrm{rad} / \mathrm{s}(\omega=2 \pi f, f=50 \mathrm{~Hz})$, capacitor $C=2 \mathrm{mF}$, dc voltage $e_{\mathrm{dc}}{ }^{*}=200 \mathrm{~V}$ and a mains voltage $U_{\mathrm{rms}}=50 \mathrm{~V}$. The resulting proportional and integral gains are $k_{\mathrm{P}}=0.4104$ and $k_{\mathrm{I}}=18.23$. A current limit of $10 \mathrm{~A}$ is used for anti-windup purposes. In the VSC and current controller it is considered: $L=L_{\mathrm{N}}=2.15 \mathrm{mH}$, $R=R_{\mathrm{N}}=0,1 \Omega$ and a current hysteresis band $H_{123 \mathrm{~N}}= \pm 0.25 \mathrm{~A}$. It should be noted that the regulator gains depends upon the mains voltage and VSC dc voltage so the controller dynamic changes with the operation conditions, unless the gains are adapted measuring those variables.

\section{System Control with a Sliding-Mode Controller}

By a regulation point of view, the output variable for this MISO system is the VSC dc voltage $e_{\mathrm{dc}}$. The input variable is a priori the control function (auxiliary switching function in Park's coordinates) $f_{\mathrm{d}}$. In order to obtain a sliding-mode controller the input-output linearization technique is applied so that the state-space model (2) is written in phase-variable canonical form (PVCF), also known as controllability canonical form (CCF), (7). In practice, the voltage regulation is performed acting in current component $i_{c \mathrm{~cd}}$, therefore 
the voltage state equation needs to be derivated to include this current. Now, the state-space model in CCF includes a new state variable, $a_{\mathrm{dc}}$.

$$
\left[\begin{array}{c}
\dot{i}_{c_{\mathrm{q}}} \\
\dot{i}_{c_{0}} \\
\dot{e}_{\mathrm{dc}} \\
\dot{a}_{\mathrm{dc}}
\end{array}\right]=\left[\begin{array}{l}
-\dot{\theta} i_{c_{\mathrm{d}}}-\frac{R}{L} i_{c_{\mathrm{q}}}-\frac{f_{\mathrm{q}}}{L} e_{\mathrm{dc}}+\frac{u_{\mathrm{q}}}{L} \\
-\frac{R+3 R_{\mathrm{N}}}{L+3 L_{\mathrm{N}}} i_{c_{0}}-\frac{f_{0}}{L+3 L_{\mathrm{N}}} e_{\mathrm{dc}}+\frac{u_{0}}{L+3 L_{\mathrm{N}}} \\
a_{\mathrm{dc}} \\
-\frac{R}{L} a_{\mathrm{dc}}-\frac{1}{C}\left(\frac{f_{\mathrm{d}}^{2}+f_{\mathrm{q}}^{2}}{L}+\frac{f_{0}^{2}}{L+3 L_{\mathrm{N}}}\right) e_{\mathrm{dc}}- \\
-\frac{R}{L C} i_{\text {load }}-\frac{1}{C} \frac{\mathrm{d} i_{\text {load }}}{\mathrm{d} t}+\frac{\dot{\theta}\left(f_{\mathrm{d}} i_{c_{\mathrm{q}}}-f_{\mathrm{q}} i_{c_{\mathrm{d}}}\right)}{C}+ \\
+\frac{f_{\mathrm{d}} u_{\mathrm{d}}+f_{\mathrm{q}} u_{\mathrm{q}}+f_{0} u_{0}}{L C}
\end{array}\right]
$$

In feedback control systems is useful to introduce error variables to ensure tracking of the references, (8).

$$
e_{i_{c_{\mathrm{q}}}}=i_{c_{\mathrm{q}}}^{*}-i_{c_{\mathrm{q}}}, e_{i_{c_{0}}}=i_{c_{0}}^{*}-i_{c_{0}}, e_{e_{\mathrm{dc}}}=e_{\mathrm{dc}}^{*}-e_{\mathrm{dc}}
$$

The sliding surface $j$ that ensures robustness of a $n$ order (in a CCF) closed-loop control system is, in general, given by a linear combination of all phase canonical state variables [6] which may be written in terms of errors $e_{i}(9)$

$$
s_{j}\left(e_{i}, t\right)=e_{n}+\sum_{i=1}^{n-1} k_{i} e_{i}=0
$$

There must be as many sliding surfaces as variables to be controlled. If equation (9) is fulfilled the original order is decrease by one, so it behaves like a (n-1) order system. Gain values are imposed to obtain a desired system dynamics. This problem is also known as the pole-placement design through state feedback. Currents $i_{c q}$ and $i_{c 0}$ have a strong relative degree of one. Their first derivates (state equations) contains the control variables $f_{\mathrm{q}}$ and $f_{0}$, so the sliding surfaces that ensure robustness of the closed-loop control systems have a proportional action, i.e, have zero-order dynamics and are expressed by (10).

$$
\left\{\begin{array}{l}
s_{1}\left(e_{i_{c_{\mathrm{q}}}}, t\right)=e_{i_{c_{\mathrm{q}}}}=\left(i_{c_{\mathrm{q}}}^{*}-i_{c_{\mathrm{q}}}\right)=0 \\
s_{2}\left(e_{i_{c_{0}}}, t\right)=e_{i_{c_{0}}}=\left(i_{c_{0}}^{*}-i_{c_{0}}\right)=0
\end{array}\right.
$$

For the VSC dc voltage the strong relative degree is two, therefore the corresponding sliding surface is expressed by (11a). This equation can be viewed as a first-order LP filter with a cutoff frequency $\omega_{c}$ or a time constant $\tau,(11 b)$.

$$
\begin{gathered}
s_{3}\left(e_{e_{\mathrm{dc}}}, e_{a_{\mathrm{dc}}}, t\right)=e_{a_{\mathrm{dc}}}+k_{e_{\mathrm{dc}}} e_{e_{\mathrm{dc}}}=\frac{\mathrm{d} e_{e_{\mathrm{dc}}}}{\mathrm{d} t}+k_{e_{\mathrm{dc}}} e_{e_{\mathrm{dc}}}=0 \\
s_{3}\left(e_{e_{\mathrm{dc}}}, e_{a_{\mathrm{dc}}}, t\right)=\frac{\mathrm{d} e_{e_{\mathrm{dc}}}}{\mathrm{d} t}+\omega_{c} e_{e_{\mathrm{dc}}}=0 \quad, \quad k_{e_{\mathrm{dc}}}=\omega_{c}=\frac{1}{\tau}
\end{gathered}
$$

By the state-space model (7), (11b) can be presented in the form,

$$
\begin{aligned}
s_{3}\left(e_{e_{\mathrm{dc}}}, e_{a_{\mathrm{dc}}}, t\right)=i_{c_{\mathrm{d}}}^{*}-i_{c_{\mathrm{d}}}= \\
=\left[\left(e_{\mathrm{dc}}^{*}-e_{\mathrm{dc}}\right)+\frac{1}{\omega_{c}}\left(\frac{\mathrm{d} e_{\mathrm{dc}}^{*}}{\mathrm{~d} t}-\frac{i_{\text {load }}-f_{\mathrm{q}} i_{c_{\mathrm{q}}}-f_{0} i_{c_{0}}}{C}\right)\right] . \\
\cdot \frac{\omega_{c} C}{f_{\mathrm{d}}}-i_{c_{\mathrm{d}}}=0
\end{aligned}
$$

The auxiliary switching functions $f_{\mathrm{d}}, f_{\mathrm{q}}$, and $f_{0}$ are equal to the values presented in [2]. For voltage control purposes $f_{\mathrm{q}}$ and $f_{0}$ are made equal to zero. Under these conditions the active reference current component $i_{c \mathrm{~d}}{ }^{*}$ is,

$$
i_{\mathrm{d}_{\mathrm{h}}}^{+}=i_{c_{\mathrm{d}}}^{*}=\left[\omega_{c}\left(e_{\mathrm{dc}}^{*}-e_{\mathrm{dc}}\right)+\frac{\mathrm{d} e_{\mathrm{dc}}^{*}}{\mathrm{~d} t}-\frac{i_{\mathrm{load}}}{C}\right] \cdot \frac{C e_{\mathrm{dc}}}{\sqrt{3 / 2} \hat{U}_{123}} .
$$

Fig. 2 shows the voltage regulation diagram with a first-order switching function. A null steady-state error is guarantee only if the closed-loop control system order is increased by adding to the original system (in CCF) (7) an integral term. The corresponding control law for this situation is,

$$
s_{j}\left(e_{i}, t\right)=k_{0} \int e_{1} \mathrm{~d} t+e_{n}+\sum_{i=1}^{n-1} k_{i} e_{i}=0 .
$$

Applying (14) in the present case, equation (15) is derived.

$$
\begin{aligned}
s_{3}\left(e_{e_{\mathrm{dc}}}, e_{a_{\mathrm{dc}}}, t\right) & =k_{0} \int e_{e_{\mathrm{dc}}} \mathrm{d} t+e_{a_{\mathrm{dc}}}+k_{e_{\mathrm{dc}}} e_{e_{\mathrm{dc}}}= \\
= & k_{0} \int e_{e_{\mathrm{dc}}} \mathrm{d} t+\frac{\mathrm{d} e_{e_{\mathrm{dc}}}}{\mathrm{d} t}+k_{e_{\mathrm{dc}}} e_{e_{\mathrm{dc}}}=0
\end{aligned}
$$

With the same reasoning taken before, the integral-differential (15) can be seen as a second-order LP filter with a natural undamped frequency $\omega_{n}$ and a damping ratio $\zeta$, so $k_{0}=\omega_{n}{ }^{2}$ and $k_{e \mathrm{dc}}=2 \zeta \omega_{n}$. The reference current component $i_{c \mathrm{~d}}{ }^{*}$ is now,

$$
\begin{aligned}
i_{\mathrm{d}_{\mathrm{h}}}^{+}=i_{c_{\mathrm{d}}}^{*} & =\left[\omega_{n}^{2} \int\left(e_{\mathrm{dc}}^{*}-e_{\mathrm{dc}}\right) \mathrm{d} t+2 \zeta \omega_{c}\left(e_{\mathrm{dc}}^{*}-e_{\mathrm{dc}}\right)+\right. \\
& \left.+\frac{\mathrm{d} e_{\mathrm{dc}}^{*}}{\mathrm{~d} t}-\frac{i_{\mathrm{load}}}{C}\right] \cdot \frac{C e_{\mathrm{dc}}}{\sqrt{3 / 2} \hat{U}_{123}}
\end{aligned}
$$

The voltage regulation diagram with a second-order switching function is presented in Fig. 3.

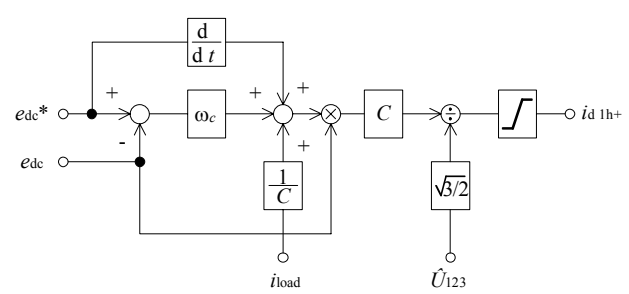

Fig. 2. Voltage regulation diagram for the sliding-mode regulator with a first-order sliding surface. 


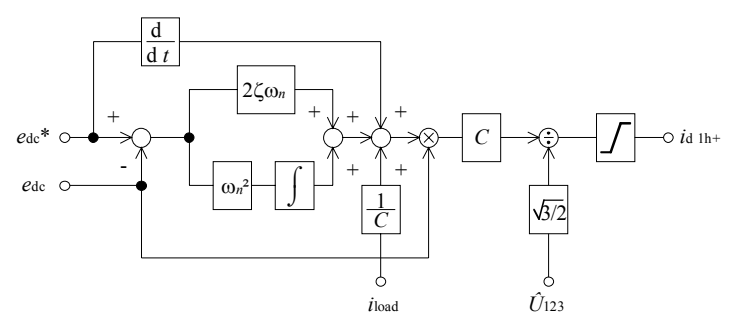

Fig. 3. Voltage regulation diagram for the sliding-mode regulator with a second-order sliding surface.

Note that there is no need for the derivate action presented in Figs. 2 and 3 under constant dc voltage operation and also for step changes in the voltage reference input so the control circuit can be simplified.

The parameters considered in the controllers syntheses for the prototype first-order sliding surface and second-order sliding surface, as presented in (13) and (16), respectively, are equal to the linear regulator. For both cases the cutoff frequency and natural undamped frequency are also the same $\omega_{c}=\omega_{n}$. In (13) it results in a time constant $\tau=1 / \omega_{c}=15.92 \mathrm{~ms}$.

The existence of the sliding-mode depends upon the availability of an equivalent VSC ac voltage value bounded by the limits $e_{\mathrm{dq} 0 \text { min }}<e_{\mathrm{dq} 0 \text { eq }}<e_{\mathrm{dq} 0 \text { max }}$ that assures always the current control.

This condition is assured by a high enough value of dc voltage $e_{\mathrm{dc}}$ initially imposed in the VSC. The hysteretic current controller in phase coordinates impose that the sliding-mode stability condition is then fulfilled (17).

$$
s_{j}\left(e_{i}, t\right) \cdot \frac{\mathrm{d} s_{j}\left(e_{i}, t\right)}{\mathrm{d} t}<0 .
$$

\section{Results}

With the three control systems proposed the VSC transient and steady state responses are presented in Figs. 4-11. The results shown in Fig. 4 were taken applying a step change of $10 \mathrm{~V}$ in the dc voltage reference $e_{\mathrm{dc}}{ }^{*}$. All the other results were obtained with a $10 \mathrm{~A}$ reactive input current step, $i_{\mathrm{q}}$, a $2.5 \mathrm{~A}$ load current step, $i_{\text {load, }}$, and a third harmonic current of zero sequence, $i_{0}$, with a current peak value of $10 / \sqrt{3} \mathrm{~A}$, Figs. 5 to 11. The load current was created by a resistive load $\left(R_{\text {load }}=80 \Omega\right)$ applied to the VSC dc side. Balanced sinusoidal voltage conditions were also considered.

Results are shown for the following cases: PI controller with a voltage reference filter (PI1) and without it (PI2); sliding-mode controller with a first-order sliding surface (SM1); sliding-mode controller with second-order sliding surface (SM2) with and without load current measurement.

The controller PI2 behaves like the sliding-mode controller SM2, Fig. 4. They present an appreciable overshoot value, greater than the value initially imposed. A voltage reference filter reduces the overshoot to the pretended value as can be seen by the result of PI1. All systems present a second order behaviour except for the case of SM1 that has a first-order response but exhibits a small static error however.

The systems are insensible to reactive current as expected, as there is no active power associated with it, Figs. 5(a)-(c) and 8(a)-(c). Active and reactive currents are almost decoupled. Mains voltage, phase and neutral currents are presented in Figs. 6 and 9. Dc bus voltage, dc bus voltage error and other variables controlled are shown in Figs. 7, 10 and 11.

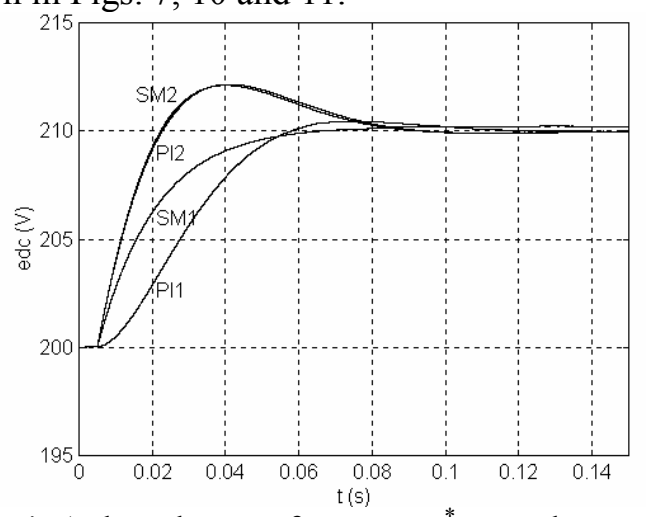

Fig. 4. A dc voltage reference $\mathrm{e}_{\mathrm{dc}}{ }^{*}$ step change in the linear controllers PI1, PI2 and sliding mode controllers SM1, SM2.

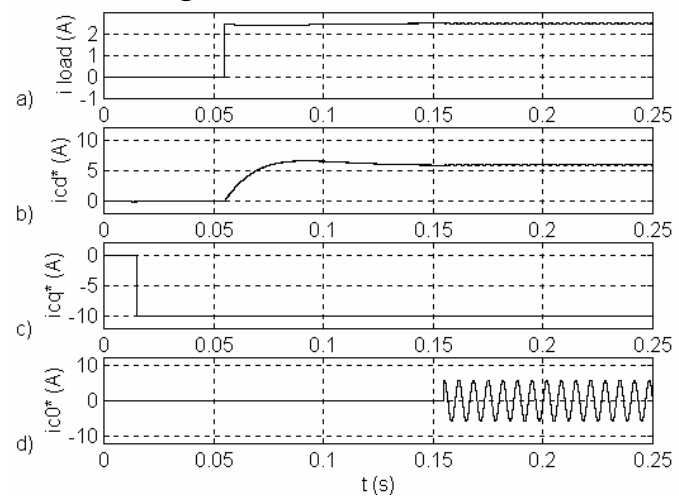

Fig. 5. Controllers PI1 and PI2 and SM2 without load current measurement: (a) Converter load current $i_{\text {load}}$;

(b) Converter active reference current $i_{c \mathrm{~d}}{ }^{*}=i_{\mathrm{d} 1 \mathrm{~h}}{ }^{+}$;

(c) Converter reactive reference current $i_{c q}{ }^{*}=i_{\mathrm{q}}$;

(d) Converter zero sequence reference current $i_{c 0}{ }^{*}=i_{0}$.

For the sake of simplicity the load current measurement in SM2 could be taken out at the expense 
of a decrease in the dynamic behaviour. Under these circumstances the sliding-mode controller behaves like a PI controller. Slow responses to load current changes are obtained with the PI1, PI2 and SM2 without load current measurement, Figs. 5(a), (b) and 6(b). Faster responses are obviously obtained in the controller SM1 and SM2 due to the load current measurement. Active reference current changes almost instantaneously and so the mains current, Figs. 8(a), (b) and 9(b).
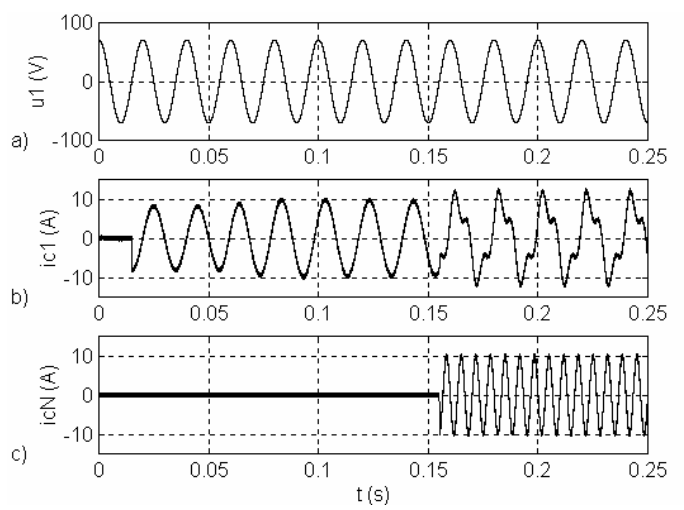

Fig. 6. Controllers PI1 and PI2 and SM2 without load current measurement: (a) Mains voltage $u_{1}$;

(b) Converter current $i_{c 1}$; (c) Converter neutral
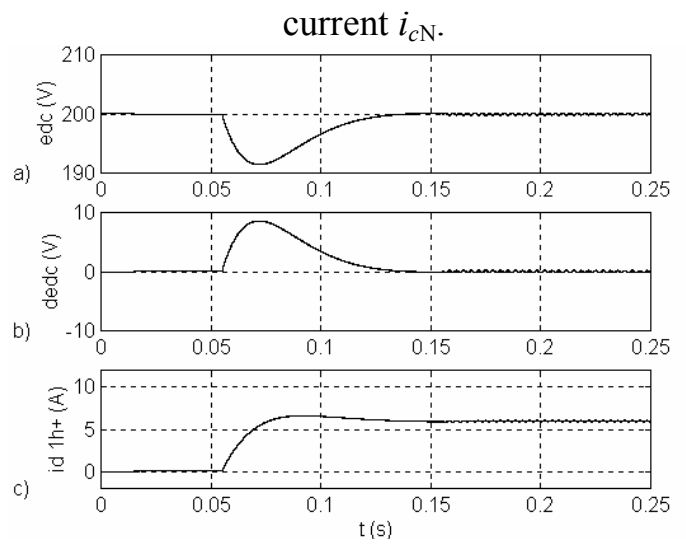

Fig. 7. Controllers PI1 and PI2 and SM2 without load current measurement: (a) Dc bus voltage $e_{\mathrm{dc}}$; (b) Dc bus voltage error $\delta e_{\mathrm{dc}}=e_{\mathrm{dc}}{ }^{*}-e_{\mathrm{dc}}$; (b) First harmonic current of positive sequence $i_{\mathrm{d} 1 \mathrm{~h}}{ }^{+}=i_{c \mathrm{~d}}{ }^{*}$.

Observing Figs. 7(a), (b) and 11(a), (b) it is clear that the steady-state error obtained is null. The absence of voltage error relatively to a large disturbance $\left(i_{\text {load }}\right)$ is due to the integral action performed by PI1 and PI2, and introduced in the sliding-mode control law of SM2. In the results of SM1 it can be seen that it exhibits a small error under steady-state conditions, Figs. 10(a) and (b).

To guarantee a null steady-state voltage error it is always necessary to used an integral action in the control laws. The load current measurement is used to improve the system response in transient states except in the case of SM1 where it is absolutely necessary otherwise high voltage errors occurs.

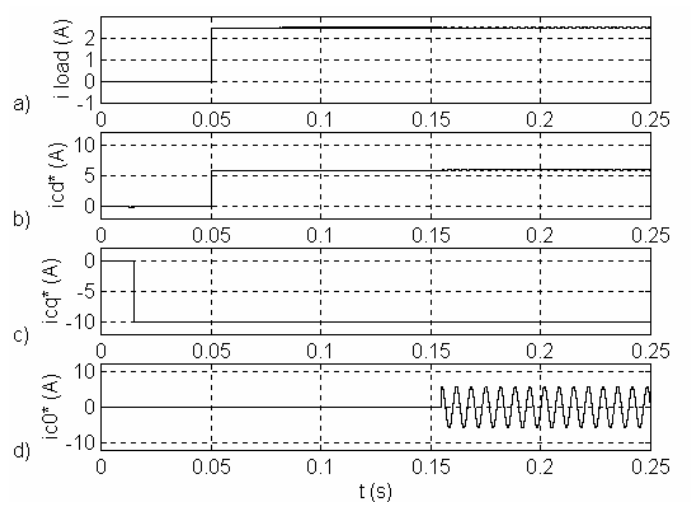

Fig. 8. Controllers SM1 and SM2: (a) Converter load current $i_{\text {load }}$; (b) Converter active reference current $i_{c \mathrm{~d}}{ }^{*}=i_{\mathrm{d} 1 \mathrm{~h}}{ }^{+} ;$(c) Converter reactive reference current $i_{c q}{ }^{*}=i_{q}$; (d) Converter zero sequence reference current $i_{c 0}{ }^{*}=i_{0}$.

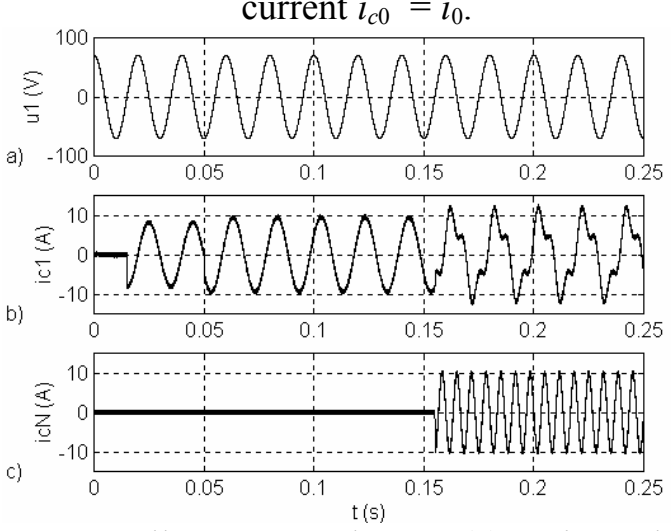

Fig. 9. Controllers SM1 and SM2: (a) Mains voltage $u_{1}$;

(b) Converter current $i_{c 1}$; (c) Converter neutral
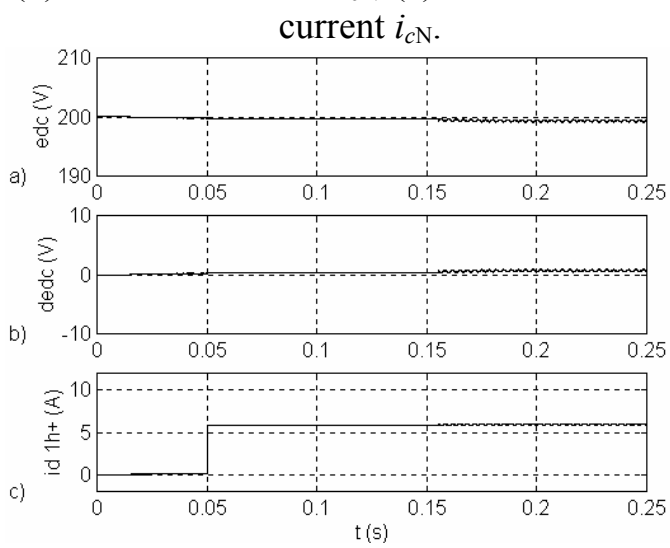

Fig. 10. Controller SM1: (a) Dc bus voltage $e_{\mathrm{dc}}$; (b) Dc bus voltage error $\delta e_{\mathrm{dc}}=e_{\mathrm{dc}}{ }^{*}-e_{\mathrm{dc}}$; (b) First harmonic current of positive sequence $i_{\mathrm{d} 1 \mathrm{~h}}{ }^{+}=i_{c \mathrm{~d}}{ }^{*}$. 

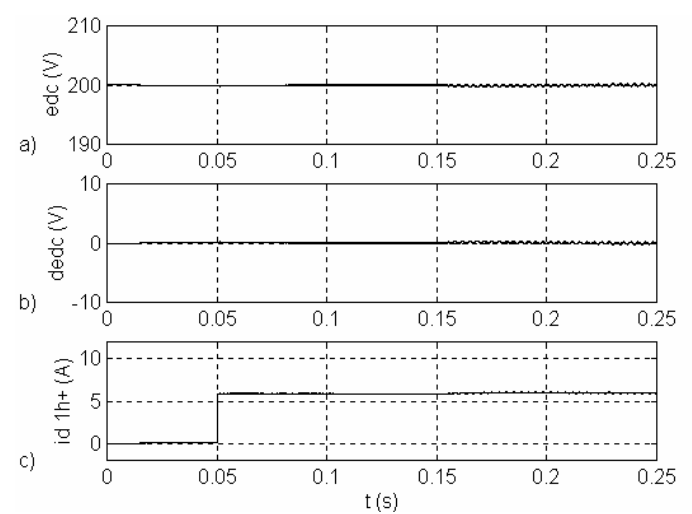

Fig. 11. Controller SM2: (a) Dc bus voltage $e_{\mathrm{dc}}$; (b) Dc bus voltage error $\delta e_{\mathrm{dc}}=e_{\mathrm{dc}}{ }^{*}-e_{\mathrm{dc}}$; (b) First harmonic current of positive sequence $i_{\mathrm{d} \mathrm{lh}}{ }^{+}=i_{c \mathrm{~d}}{ }^{*}$.

As stated before sliding-mode regulator can be simplified taking off the derivate action in voltage reference and load current measurement. Further simplifications could be performed like the mains voltage measurement, however the dynamic behaviour and robustness to mains voltage variations could not be guaranteed and it will depend upon the operation point.

To obtain a null steady-state voltage error and for a constant dc voltage operation the PI2 is the best choice. There's only need for one voltage measurement and the control circuit is the simplest and easy to implement. It is also the best by the point of view of mains current smoothness avoiding fast changes and consequently voltage drops in mains impedance's. This is useful in AF's, UPFC's and NCC's since they operate under constant dc voltage and voltage regulation is not the primary goal. Reference voltage changes and parameters variation, like mains voltage and capacitor degradation, does not affect the steady-state error but change the dynamic behaviour as proportional and integral gains depend on it.

For a null voltage error and the highest possible dynamic behaviour the SM2 is obviously the preferred control circuit at the expense of an increased complexity. In this case two additional measurements are needed compared with PI1 and PI2. Also product and division operations are required which are more difficult to implement with analogue circuits. The PWM rectifiers can benefit from this high dynamic behaviour controller.

\section{Conclusions}

In this paper three different voltage regulation systems were presented for a three-phase four-wire system based on a four-leg VSC.
A complete dc side model was presented enabling the synthesis of a PI controller and sliding-mode controllers for voltage regulation purposes. All models presented proved to be valid and stable systems were also achieved. The results obtained establish the validity of the models used and simplifications performed.

Steady-state and transient performance issues were analysed. It was concluded that there is a trade-off between controller simplicity and steady-state error, dynamic behaviour and system robustness.

Also, there isn't a unique and global control choice for the voltage regulation problem. It depends on the acceptable errors and performance needed. Therefore, the best overall regulator cannot be stated. The controller advisable depends upon the application (e.g. APF's, UPQC's and PWM rectifiers). The four-leg VSC with the voltage regulation schemes presented can be used in all applications with or without neutral current control.

\section{References:}

[1] L. A. Pittorino, J. A. du Toit, and J. H. R. Enslin, Evaluation of converter topologies and controllers for power quality compensators under unbalanced conditions, in Proc. PESC'97 Conf., ISBN 0-7803-3840-5, vol. 2, pp. 1127-1133, 1997.

[2] V. Soares and P. Verdelho, Active power filter with neutral current compensation based on the extension of the instantaneous active and reactive current component $i_{d}-i_{q}$ method, in Proc. EPE'99 Conf., CD-ROM, ISBN 90-75815-04-02, pp. P1-P10, 1999.

[3] P. Verdelho, Modelling and simulation of a fundamental reactive power and unbalanced currents compensator, in Proc. ISIE'99, Conf., pp. 843-848, 1999.

[4] V. Soares and P. Verdelho, Voltage Regulation System Design for the Four-Leg Converter, in Proc. EPE'03 Conf., CD-ROM, ISBN 90-75815-07-7, pp. P1-P10, 2003.

[5] V. Soares, P. Verdelho and G. Marques, An Instantaneous Active and Reactive Current Component Method for Active Filters, IEEE Trans. on Power Electronics, vol. 15, no. 4, pp. 660-669, July, 2000.

[6] J. Hung, W. Gao, and J. Hung, Variable Structure Control: A survey, IEEE Trans. on Industrial Electronics, vol. 40, pp. 2-22, Feb. 1993. 